\title{
A UNIVERSAL DIAGRAM PROPERTY OF MINIMAL NORMAL EXTENSIONS ${ }^{1}$
}

\author{
JOHN W. BUNCE
}

\begin{abstract}
We give a universal diagram property which characterizes, up to algebraic equivalence, the minimal normal extension of a subnormal operator.
\end{abstract}

1. Introduction. An operator $S$ on a Hilbert space $\mathcal{H}$ is called subnormal if there exists a normal operator $N$ on a larger Hilbert space $\mathscr{K} \supseteq \mathcal{H}$ such that $S=N \mid \mathcal{H}$. Such a normal operator $N$ is called a normal extension of $S$. If, in addition, $\mathcal{K}$ is the smallest closed subspace of $\mathscr{K}$ containing $\mathcal{H}$ and reducing $N$, then $N$ is called the minimal normal extension of $S$. P. R. Halmos [8] introduced subnormal operators and showed that there is always an essentially unique minimal normal extension of a subnormal operator.

In what is still the basic paper on subnormal operators, J. Bram showed, among many other things, that if $\theta$ is a *-isomorphism between a $C^{*}$-algebra $\mathbb{Q}_{1} \subseteq B\left(\mathcal{H}_{1}\right)$ and a $C^{*}$-algebra $\mathbb{Q}_{2} \subseteq B\left(\mathcal{F}_{2}\right)$, then $A \in \mathbb{Q}_{1}$ is subnormal if and only if $\theta(A)$ is subnormal $[3, \mathrm{p}$. 80]. Thus the concept of subnormality is actually an algebraic property, not a spatial property. An explicit algebraic characterization of subnormality was given in [4, Proposition 1]. This characterization makes obvious the fact that if $\theta: \mathbb{Q}_{1} \rightarrow \mathbb{Q}_{2}$ is a ${ }^{*}$-homomorphism then $\theta(A)$ is subnormal if $A$ is subnormal. The purpose of this paper is to give an algebraic characterization of the minimal normal extension of a subnormal operator. We characterize the minimal normal extension as the universal object for certain diagrams of completely positive maps. The methods used in this paper are essentially the methods of Bram applied to the Stinespring decomposition of various completely positive maps. In $\$ 3$ we remark that similar $C^{*}$-algebraic characterizations can be given for the minimal strong unitary dilation and minimal coisometric extension of a contraction.

2. The universal property of minimal normal extensions. We refer the reader to [2, Chapter 1] for the definition and basic facts concerning completely positive maps and their Stinespring decompositions. For a subnormal operator $S \in B(\mathcal{H})$ let $N \in B(\mathscr{K})$ be the minimal normal extension of $S$. Let $P$ be

Received by the editors March 28, 1977 and, in revised form, July 26, 1977.

AMS (MOS) subject classifications (1970). Primary 47B20; Secondary 46L05.

Key words and phrases. Subnormal operators, minimal normal extensions, algebraic equivalence, completely positive maps, Stinespring decomposition.

${ }^{1}$ Research partially supported by the National Science Foundation. 
the orthogonal projection of $\mathscr{K}$ onto $\mathcal{H}$. We shall henceforth call the pair $(N, P)$ the minimal normal extension of $S$.

For $A$ an element of any $C^{*}$-algebra with identity we denote by $C^{*}(A)$ the $C^{*}$-algebra generated by $A$ and the identity. If $\varphi: \mathbb{Q}_{1} \rightarrow \mathbb{Q}_{2}$ is a completely positive map between $C^{*}$-algebras and $A \in \mathbb{Q}_{1}$, we say that $\varphi$ is $A$-multiplicative if $\varphi\left(A^{*} A\right)=\varphi\left(A^{*}\right) \varphi(A)$.

THEOREM 1. Let $S \in B(\mathcal{H C})$ be a subnormal operator with minimal normal extension $(N, P)$. Define $\theta: C^{*}(N) \rightarrow C^{*}(S)$ by $\theta(A)=P A \mid \mathcal{H}$. Then $\theta$ is a unital completely positive $N$-multiplicative map with $\theta(N)=S$. Moreover, if $M$ is a normal element of some $C^{*}$-algebra and $\varphi: C^{*}(M) \rightarrow C^{*}(S)$ is a unital completely positive $M$-multiplicative map with $\varphi(M)=S$, then there exists a *-homomorphism $\rho: C^{*}(M) \rightarrow C^{*}(N)$ with $\rho(M)=N$.

Proof. It is clear that $\theta: B(\mathscr{K}) \rightarrow B(\mathcal{H})$ given by $\theta(A)=P A \mid \mathcal{H}$ is completely positive and $\theta(N)=S$. Since $S^{*}=P N^{*} \mid \mathcal{H}, \theta\left(N^{* i} N^{j}\right)=S^{* i} S^{j}$ for all $i, j \geqslant 0$. Thus $\theta\left(\sum \alpha_{i j} N^{* i} N^{j}\right)$ is in $C^{*}(S)$, and $\theta$ maps any element of the *-algebra generated by $N$ into $C^{*}(S)$. It follows that $\theta$ maps $C^{*}(N)$ into $C^{*}(S)$.

Suppose now that $\varphi: C^{*}(M) \rightarrow C^{*}(S)$ is a unital completely positive $M$-multiplicative map with $\varphi(M)=S$. Let $\varphi=V^{*} \pi V$ be the Stinespring decomposition of $\varphi$; that is, $\pi: C^{*}(M) \rightarrow B\left(\mathcal{H}_{\pi}\right)$ is a *-representation of $C^{*}(M), V: \mathcal{H} \rightarrow \mathcal{H}_{\pi}$ is an isometry, $\varphi(A)=V^{*} \pi(A) V$ for all $A$ in $C^{*}(M)$, and $\mathcal{H}_{\pi}=\left[\pi(A) V x: A \in C^{*}(M), x \in \mathcal{H}\right]$. Since $\varphi\left(M^{*} M\right)=\varphi\left(M^{*}\right) \varphi(M)$, $V(\mathcal{H})$ is an invariant subspace for $\pi(M)([6$, Remark 4.11] or [5, Remark 4.3]) and $\varphi(A M)=\varphi(A) \varphi(M)$ for all $A \in C^{*}(M)([6$, Theorem 4.1] or [10, p. 268]). Thus $\mathcal{H}_{\pi}=\left[\pi\left(M^{* i}\right) V x: i \geqslant 0, x \in \mathcal{H}\right]$. We define a linear transformation $W$ from $\mathcal{H}_{\pi}$ into $\mathcal{H}$ by setting

$$
W\left(\sum_{i=0}^{n} \pi\left(M^{* i}\right) V x_{i}\right)=\sum_{i=0}^{n} N^{* i} x_{i}
$$

Then (as in the proof that minimal normal extensions are unique [9, Solution 155]) we compute:

$$
\begin{aligned}
\left\|\sum_{i=0}^{n} N^{* i} x_{i}\right\|^{2} & =\sum_{i, j}\left(S^{j} x_{i}, S^{i} x_{j}\right)=\sum\left(S^{* i} S^{j} x_{i}, x_{j}\right)=\sum\left(\varphi\left(M^{* i} M^{j}\right) x_{i}, x_{j}\right) \\
& =\sum\left(\pi\left(M^{* i} M^{j}\right) V x_{i}, V x_{j}\right)=\left\|\sum_{i=0}^{n} \pi\left(M^{* i}\right) V x_{i}\right\|^{2} .
\end{aligned}
$$

Hence $W$ can be extended to be a unitary transformation from $\mathcal{K}_{\pi}$ to $\mathcal{K}$, and clearly $W \pi(M)=N W$. Now define $\rho: C^{*}(M) \rightarrow C^{*}(N)$ by $\rho(A)=$ $W \pi(A) W^{*}$. Q.E.D.

Theorem 1 shows that $\theta: C^{*}(N) \rightarrow C^{*}(S)$ can be characterized, up to algebraic equivalence, as the universal object for diagrams $\varphi: C^{*}(M) \rightarrow$ $C^{*}(S)$ of unital completely positive $M$-multiplicative maps with $\varphi(M)=S$. The minimal normal extension can thus be characterized (up to algebraic 
equivalence) in a nonspatial manner. Theorem 1 easily yields the following corollary, which was conjectured in [1, Problem 2] and first proved in [4, Corollary 2]. The present proof is perhaps the one envisioned in [1].

COROLlaRy 2. Let $S \in B(\mathcal{H C})$ be subnormal with minimal normal extension $N$ and let $\pi: C^{*}(S) \rightarrow B\left(\mathcal{H}_{\pi}\right)$ be $a^{*}$-representation. If $M$ is the minimal normal extension of $\pi(S)$, then there is a $a^{*}$-homomorphism $\rho: C^{*}(N) \rightarrow C^{*}(M)$ with $\rho(N)=M$.

Proof. Let $\theta: C^{*}(N) \rightarrow C^{*}(S)$ and $\psi: C^{*}(M) \rightarrow C^{*}(\pi(S))$ be the completely positive compression maps defined in Theorem 1 . Then $\pi \circ \theta: C^{*}(N)$ $\rightarrow C^{*}(\pi(S))$ is completely positive and $N$-multiplicative. Theorem 1 then asserts the existence of the homomorphism $\rho$.

If $S$ is subnormal with minimal normal extension $N$ and $\pi$ is a *-representation of $C^{*}(S)$, then Corollary 2 shows that there is an associated *-representation $\tilde{\pi}$ of $C^{*}(N)$ such that $\tilde{\pi}(N)$ is the minimal normal extension of $\pi(S)$. The following theorem shows that the correspondence $\pi \rightarrow \tilde{\pi}$ is functorial.

THEOREM 3. Let $S$ be subnormal with minimal normal extension $(N, \theta)$ and let $\pi: C^{*}(S) \rightarrow B\left(\mathcal{H}_{\pi}\right)$ be a ${ }^{*}$-representation. If $\pi \circ \theta$ has Stinespring decomposition $V^{*} \tilde{\pi} V$, then $\left(\tilde{\pi}(N), V V^{*}\right)$ is the minimal normal extension of $\pi(S)$. Moreover, if $\pi_{1}$ and $\pi_{2}$ are two ${ }^{*}$-representations of $C^{*}(S)$ and $T \pi_{1}(A)=$ $\pi_{2}(A) T$ for all $A$ in $C^{*}(S)$, then there is an operator $\tilde{T}$ such that $\tilde{T} \tilde{\pi}_{1}(A)=$ $\tilde{\pi}_{2}(A) \tilde{T}$ for all $A$ in $C^{*}(N)$.

Proof. Let $\pi \circ \theta$ have Stinespring decomposition $V^{*} \tilde{\pi} V$, where $\tilde{\pi}: C^{*}(N)$ $\rightarrow B\left(\mathcal{H}_{\tilde{\pi}}\right)$ is a ${ }^{*}$-representation and $V: \mathcal{H}_{\pi} \rightarrow \mathcal{H}_{\tilde{\pi}}$ is an isometry. Then $V^{*} \tilde{\pi}(N) V=\pi(\theta(N))=\pi(S)$. Since $\pi \circ \theta$ is $N$-multiplicative, $V\left(\mathcal{H}_{\pi}\right)$ is invariant for $\tilde{\pi}(N)$, and since

$$
\begin{aligned}
\mathcal{H}_{\tilde{\pi}} & =\left[\tilde{\pi}(A) V x: A \in C^{*}(N), x \in \mathcal{H}_{\pi}\right] \\
& =\left[\tilde{\pi}\left(N^{* i}\right) V x: i \geqslant 0, x \in \mathcal{H}_{\pi}\right]
\end{aligned}
$$

it is clear that $\tilde{\pi}(N)$ can be identified with the minimal normal extension of $\pi(S)$.

Let $\pi_{1}$ and $\pi_{2}$ be two such *-representations of $C^{*}(S)$ and let $T: \mathcal{H}_{\pi_{1}} \rightarrow \mathcal{H}_{\pi_{2}}$ be such that $T \pi_{1}(A)=\pi_{2}(A) T$ for all $A$ in $C^{*}(S)$. The rest of the theorem follows from [2, Theorem 1.3.1\}-let the $V$ of [2, Theorem 1.3.1] be $V_{1} \oplus V_{2}$ and let $\mathscr{B}$ be $\left(\tilde{\pi}_{1} \oplus \tilde{\pi}_{2}\right)\left(C^{*}(N)\right)$.

Since Theorem 1 gives an algebraic characterization of the minimal normal extension $N$ of a subnormal operator $S$, it is natural to ask if there is a nonspatial way to construct $N$. In the rest of this section we outline such a construction, using the $C^{*}(S)$-modules of [11] and [12].

Let $S$ be an element of a $C^{*}$-algebra $Q$ with identity. We will say that $S$ is subnormal if for all finite sets $B_{0}, B_{1}, \ldots, B_{n}$ in $\mathcal{Q}$ we have

$$
\sum_{i, j=0}^{n} B_{i}^{*} S^{* j} S^{i} B_{j} \geqslant 0
$$


By [4, Proposition 1 and Remark 1], if $Q$ is identified as a $C^{*}$-subalgebra of $B(\mathcal{H})$ for some Hilbert space $\mathcal{H}$, then $S$ is subnormal in the usual (spatial) sense if and only if (1) is satisfied. Theorem 1 of [3] and the method of proof of [4, Proposition 1] show that if $S \in \mathbb{Q}$ is subnormal then for all finite sets $B_{0}, B_{1}, B_{2}, \ldots, B_{n}$ in $Q$ we have

$$
\sum_{i, j=0}^{n} B_{i}^{*} S^{* j} S^{*} S S^{i} B_{j} \leqslant\|S\|^{2} \sum_{i, j=0}^{n} B_{i}^{*} S^{* j} S^{i} B_{j}
$$

There should be a direct $C^{*}$-algebraic proof that (1) implies (2) without using Theorem 1 of [3], but we have been unable to find such a proof. If $S$ is a subnormal operator in some $C^{*}$-algebra, we will say that $(N, \theta)$ is the minimal normal extension of $S$ if $\theta: C^{*}(N) \rightarrow C^{*}(S)$ is a universal object for diagrams $\psi: C^{*}(M) \rightarrow C^{*}(S)$ of unital completely positive $M$-multiplicative maps which take $M$ to $S$, where $M$ is a normal operator.

Let $S$ be a subnormal operator in some $C^{*}$-algebra. Let $X_{0}$ be the set of all finitely nonzero sequences $\left(A_{j}\right)_{j=0}^{\infty}$ with each $A_{j}$ in $C^{*}(S)$. We make $X_{0}$ into a $C^{*}(S)$-module by defining $\left(A_{j}\right) \cdot B=\left(A_{j} B\right)$ and define a positive conjugatebilinear $C^{*}(S)$-valued form on $X_{0}$ by

$$
\left\langle\left(A_{j}\right),\left(B_{j}\right)\right\rangle=\sum B_{j}^{*} S^{* i} S^{j} A_{i} .
$$

This form satisfies (i), (iii) and (iv) of [11, Definition 2.1]. Let $X$ be the Hilbert $C^{*}(S)$-module obtained from $X_{0}$ by factoring out the vectors of norm zero and then taking the completion (see [11]).

Define a linear operator $N$ on $X_{0}$ by $N\left(\left(A_{j}\right)\right)=\left(S A_{j}\right)$. Since $S$ satisfies (2), $N$ is bounded and determines a bounded linear operator $N$ on $X$. If we define $N^{*}$ on $X_{0}$ by

$$
N^{*}\left(A_{0}, A_{1}, A_{2}, \ldots\right)=\left(0, A_{0}, A_{1}, A_{2}, \ldots\right),
$$

then $N^{*}$ is the formal adjoint of $N\left[11\right.$, p. 447] and $N^{*} N=N N^{*}$. Let $\hat{I}$ be the element $(I, 0,0,0, \ldots)$ of $X_{0}$ and let $\theta: C^{*}(N) \rightarrow C^{*}(S)$ be given by $\theta(A)=$ $\langle A \hat{I}, \hat{I}\rangle$. Then $\theta$ is a unital completely positive $N$-multiplicative map and $\theta(N)=S$.

We sketch the proof that $\theta: C^{*}(N) \rightarrow C^{*}(S)$ is a universal object for the appropriate diagrams. Let $M$ be a normal element of some $C^{*}$-algebra and let $\psi: C^{*}(M) \rightarrow C^{*}(S)$ be a unital completely positive $M$-multiplicative map with $\psi(M)=S$. Then by Paschke's decomposition theorem for completely positive maps [11, Theorem 5.2] there is a Hilbert $C^{*}(S)$-module $Y$, a *-representation $\pi$ of $C^{*}(M)$ on $Y$, and an element $e \in Y$ such that $\psi(C)=$ $\langle\pi(C) e, e\rangle$ for all $C \in C^{*}(M)$ and $Y=\left[\pi(C)(e \cdot A): C \in C^{*}(M), A \in\right.$ $C^{*}(S)$ ]. Since $\psi$ is $M$-multiplicative, $\pi(M)$ leaves the set $e \cdot C^{*}(S)$ invariant; thus $Y=\left[\pi\left(M^{* i}\right)(e \cdot A): i \geqslant 0, A \in C^{*}(S)\right]$. Then, as in [9, p. 154], defining $W: Y \rightarrow X$ by $W\left(\sum \pi\left(M^{* i}\right)\left(e \cdot A_{i}\right)\right)=\sum N^{* i}\left(\hat{I} \cdot A_{i}\right)$ yields an isometry from $Y$ onto $X$ whose inverse $W^{-1}: X \rightarrow Y$ satisfies $\langle W y, x\rangle=\left\langle y, W^{-1} x\right\rangle$ for all $x \in X, y \in Y$, and $N W=W \pi(M)$. The map $\rho: C^{*}(M) \rightarrow C^{*}(N)$ given by $\rho(C)=W \pi(C) W^{-1}$ is then a ${ }^{*}$-homomorphism with $\rho(M)=N$; thus $\theta$ : 
$C^{*}(N) \rightarrow C^{*}(S)$ is a universal object for the appropriate diagrams.

If $\pi: C^{*}(S) \rightarrow B\left(\mathcal{H}_{\pi}\right)$ is a ${ }^{*}$-representation, then the representation $\tilde{\pi}$ of $C^{*}(N)$ discussed in Theorem 3 is precisely the representation obtained by inducing $\pi$ from $C^{*}(S)$ to $C^{*}(N)$ via $X$ ( see [12, Theorem 5.1 and Definition 5.2]). The statement in Theorem 3 concerning the lifting of intertwining operators is just [12, Theorem 5.3].

3. Universal diagram properties of other types of dilations. In this section we state, without proof, the characterization (up to algebraic equivalence) of the minimal strong unitary dilation and coisometric extension of a contraction in terms of being the universal object for certain diagrams. We refer the reader to [7] for the definitions and properties of the minimal strong unitary dilation and coisometric extension of a contraction. Since in most cases the minimal strong unitary dilation is a bilateral shift, the results for the minimal normal extension of a subnormal operator are of more interest than the results of this section.

Let $T \in B(\mathcal{H})$ with $\|T\| \leqslant 1$ and let $U \in B(\mathcal{K})$ be the minimal strong unitary dilation of $T$, with $P$ the projection of $\mathcal{K}$ onto $\mathcal{H}$. Define $\theta$ : $C^{*}(U) \rightarrow C^{*}(T)$ by $\theta(A)=P A \mid \mathcal{H}$. Then $\theta$ is completely positive, $\theta\left(U^{i}\right)=$ $T^{i}$ for all $i \geqslant 0$ and methods similar to those of section two show that $\theta$ : $C^{*}(U) \rightarrow C^{*}(T)$ is a universal object for diagrams $\psi: C^{*}(W) \rightarrow C^{*}(T)$ of unital completely positive maps with $\psi\left(W^{i}\right)=T^{i}$ for $i \geqslant 0$ and $W$ unitary. The analog of Theorem 3 also holds in this case. If $T$ is assumed to be a contraction operator in an abstractly given $C^{*}$-algebra, then, as outlined in section two for the case of the normal extension, the universal object $\theta$ : $C^{*}(U) \rightarrow C^{*}(T)$ can be constructed using $C^{*}(T)$-modules.

For a contraction $T \in B(\mathcal{H})$ with minimal coisometric extension $U \in$ $B(\mathcal{K})$ and projection $P$ from $\mathscr{K}$ onto $\mathcal{H}$, let $\theta: C^{*}(U) \rightarrow C^{*}(T)$ be given by $\theta(A)=P A \mid \mathcal{H}$. Then $\theta: C^{*}(U) \rightarrow C^{*}(T)$ is a universal object for diagrams $\psi$ : $C^{*}(W) \rightarrow C^{*}(T)$ of unital completely positive $W$-multiplicative maps with $\psi(W)=T$ and $W$ a coisometry. Again, a version of Theorem 3 is true in this case and $\theta: C^{*}(U) \rightarrow C^{*}(T)$ can be constructed in a space-free manner.

\section{REFERENCES}

1. M. B. Abrahamse and R. G. Douglas, A class of subnormal operators related to multiplyconnected domains, Advances in Math. 19 (1976), 106-148.

2. W. B. Arveson, Subalgebras of $C^{*}$-algebras, Acta Math. 123 (1969), 141-224.

3. J. Bram, Subnormal operators, Duke Math. J. 22 (1955), 75-94.

4. J. W. Bunce and J. A. Deddens, On the normal spectrum of a subnormal operator, Proc. Amer. Math. Soc. 63 (1977), 107-110.

5. J. W. Bunce and N. Salinas, Completely positive maps on $C^{*}$-algebras and the left matricial spectra of an operator, Duke Math. J. 43 (1976), 747-774.

6. M. D. Choi, Positive linear maps on $C^{*}$-algebras, Dissertation, Univ. of Toronto, 1972.

7. P. A. Fillmore, Notes on operator theory, Van Nostrand, Princeton, N. J., 1970.

8. P. R. Halmos, Normal dilations and extensions of operators, Summa Brasil Math. 2 (1950), $125-134$.

9. P. R. Halmos, A Hilbert space problem book, Van Nostrand, Princeton, N. J., 1967. 
10. T. W. Palmer, Characterizations of *-homomorphisms and expectations, Proc. Amer. Math. Soc. 45 (1974), 265-272.

11. W. L. Puschke, Inner product modules over $B^{*}$-algebras, Trans. Amer. Math. Soc. 18 (1973), 443-468.

12. M. A. Rieffel, Induced representations of $C^{*}$-algebras, Advances in Math. 13 (1974), 176-257.

Departmient of Mathematics, University of Kansas, Lawreancb, Kansas 66045 